\title{
Cost/Revenue Trade-off of Small Cell Networks in the Millimetre Wavebands
}

\author{
Emanuel Teixeira, Fernando J. Velez \\ Instituto das Telecomunicações - DEM \\ Universidade da Beira Interior, Faculdade de Engenharia \\ 6201-001 Covilhã, Portugal \\ fjv@ubi.pt, emanuelt@ubi.pt
}

\begin{abstract}
In this work, we identify and discuss the potentialities of mobile cellular communications in the millimetre wavebands, showing that very high bit/data rates can be supported in small cells with short-range coverage. We study the behaviour of the carrier-to-noise-plus-interference ratio with the coverage distance (and the actual distance from users to the eNBs) in actual environments and the respective analysis of the impact in cellular planning and optimization process. We assess the equivalent supported throughput within cells with reuse pattern $K=2$, assuming, in this preliminary phase, the use of LTE. In terms of cell coverage and the computation of interference, LoS propagation models have been considered at the $28,38,60$ and 73 GHz frequency bands. From these analytical computations, we conclude that, at $28 \mathrm{GHz}$, although lower system capacity is achieved for very short coverage distances of the order of $25 \mathrm{~m}$, in comparison to the $73 \mathrm{GHz}$ frequency band, there is an enhancement in the supported throughput for longer coverage ranges, and it is clearly more favourable for the lowest frequency band. Owing to the additional attenuation of oxygen, the $60 \mathrm{GHz}$ frequency band is more challenging, as the lowest values of the cochannel interference due to the additional oxygen attenuation originate higher values for the supported throughput, even for lower values of the reuse pattern. Based on these results, costs and revenues are studied. Revenues are proportional to the supported throughput and the profit is generally a declining function with cell radii. The highest profit corresponds to the shortest cell radii, up to $80 \mathrm{~m}$, and the best results occur for the $28 \mathrm{GHz}$ band.
\end{abstract}

Keywords: Millimetre Waves $(\mathrm{mm} W), 4 G$ evolution to $5 G, L T E, L o S$ propagation models, interference, CNIR, LTE-U.

\section{INTRODUCTION}

The millimetre wavebands can provide high bit rates in short range applications while being supported by much smaller antennas. However, in general, they suffer from higher path loss but may also have the advantage of additional oxygen absorption to reduce interference, e.g., in the $60 \mathrm{GHz}$ frequency band, as described in [1], [2]. In real environments, cellular mobile connections are simultaneously affected by noise and cochannel interference. Moreover, in the cellular planning process, the understanding of the variation of the carrier-to-noise-plusinterference ratio (CNIR) for mobile communications are of utmost importance. To study the joint contribution of all these factors in the optimization process, this paper performs a comparison of the CNIR and the equivalent supported throughput among different frequency bands for regular shaped cellular topologies such as the Manhattan grid topologies or linear topologies, like main roads or highways, as shown in
Figure 1. In fact, it is straightforward to show that, in the downlink (DL), the worst-case bounds for CNIR from a Manhattan grid topology shown in Figure 2 are like the worstcase bounds from a linear cellular topology. In order to simplify the computations, in this phase, we assume the linear topology from Figure 1, with reuse pattern two, i.e., $K=2$ [3].

The cell coverage and frequency reuse trade-off is analysed by considering the appropriate propagation models, in order to examine the influence of oxygen in the path loss at $60 \mathrm{GHz}$. The CNIR is studied in detail, as well as the underlying throughput in other frequency bands at the millimetre wavebands. These future systems will use $5 \mathrm{G}$ air interfaces that will consider, e.g., Filter Bank Modulation Carrier (FBMC). However, in the nearfuture LTE Unlicensed (at 3.5 and $5 \mathrm{GHz}$ ) is still considering OFDMA in the DL, with maximum bandwidths of $20 \mathrm{MHz}$. As a working assumption, we are considering the use of OFDMA, as in the DL of LTE Unlicensed (LTE-U), and bandwidth of 20 MHz. Hence, 100 resource blocks (RBs) are assumed. The propagation exponent is $\gamma=2.1$ for $28 \mathrm{GHz}$, and $\gamma=2.3$ for 40 $\mathrm{GHz}, 60 \mathrm{GHz}$ and $73 \mathrm{GHz}$.

By considering the results for the system capacity arising from this analysis, one examines the cost/ revenue trade-off. Costs depend on the prices for the spectrum license and equipment, operation and maintenance, as well as on the number of cells and cell radius, and take advantage of unlicensed bands.

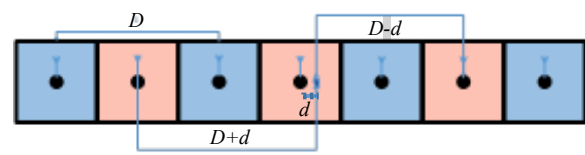

Fig. 1. Linear topology with cigar-shaped cells, with reuse pattern 2, considered in the analysis of CNIR in the millimetre wavebands.

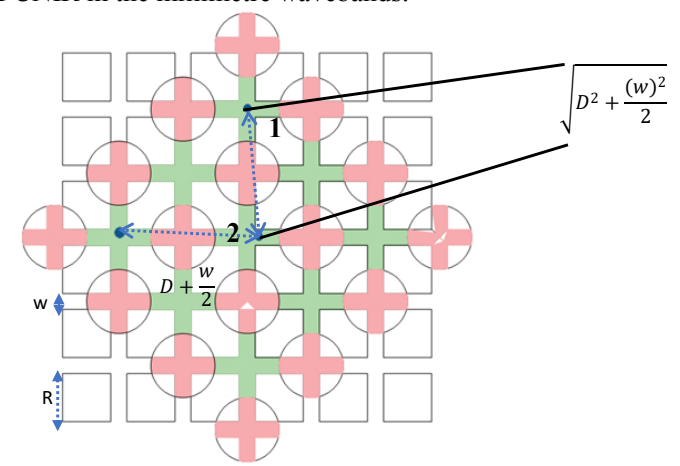

Fig. 2. Manhattan Topology (position 1 and 2).

This work has been partially supported and funded by CREaTION, COST CA 15104, ECOOP, UID/EEA/50008/ 2013 and Bolsa BID/ICIFE/Santander Universidades-UBI/2017. 
Revenues depend on the price per MB and on the supported throughput.

The remainder of the paper is organized as follows. Section II starts by presenting path loss models in millimetre wavebands, and then addresses the influence of interferers in the system, for the Manhattan and linear topologies. Section III presents the study of the system in terms of the CNIR and PHY Throughput. In Section IV, simulation and analysis of the results for CNIR and PHY Throughput are continued to study system Capacity. While in Section V, a cost/revenue model is studied in order to optimise the system. Finally, conclusions are drawn in Section VI.

\section{CARRIER-TO-INTERFERENCE RATIO IN THE MILLIMETRE WAVEBANDS}

In order to describe the behaviour related to the losses in the millimetre wavebands in Line-of-Sight (LoS), the path loss is defined by the following equation [4], [5], [6], [7]:

$$
P L[d B](d)=20 \log _{10}\left(\frac{4 \pi d_{0}}{\lambda}\right)+10 \bar{n} \log _{10}\left(\frac{d}{d_{0}}\right)+X_{\sigma}, d \geq d_{0}
$$

Where $X_{\sigma}$ is the typical log-normal random variable with 0 $\mathrm{dB}$ mean and standard deviation $\sigma$ in decibels to model shadow fading, and $d_{0}=1 \mathrm{~m}$. Therefore:

$$
P L_{L o S}[d B](d)=20 \log _{10}\left(\frac{4 \pi}{\lambda}\right)+10 \bar{n} \log _{10}(d)+X_{\sigma}, d \geq 1 m
$$

As described in [1], [8], [9] in the specific $60 \mathrm{GHz}$ frequency band, there is high oxygen and rain attenuation.

In the analysis of the DL carrier-to-interference ratio, we analyse the behaviour of interference between the interference cases where the user equipment (UE) is either at the cell edge (position 1) or at the edge of the "street crossing" (position 2). Equations (3) and (4) define the carrier-to-interference ratio for the respective cases, as follows:

$$
\begin{gathered}
\frac{C}{I}=\frac{P_{R}(R)}{P_{R}(D-R)}=\frac{1}{\left(\left(\frac{D}{R}-1\right)^{-\gamma} \cdot 10^{\frac{-\gamma_{0} R}{10}}+\left(\frac{D}{R}+1\right)^{-\gamma}\right) 10^{\frac{-\gamma_{0} D}{10}}} \\
\frac{C}{I}=\frac{\left(\frac{w}{2 R}\right)^{-\gamma}}{\left(\frac{D}{R}-\frac{w}{2 R}\right)^{-\gamma}+\left(\frac{D}{R}+\frac{w}{2 R}\right)^{-\gamma}+2\left(\sqrt{\left(\frac{D}{R}\right)^{2}+\left(\frac{w}{2 R}\right)^{2}}\right)^{-\gamma}}
\end{gathered}
$$

where $R$ is the cell length, $D$ is the reuse distance, and $w$ is the street width. Figure 3 shows the comparison of the variation of $C / I$ with $R$ between the two cases (user equipment at position 1, i.e., cell edge, and at position 2 , i.e., street crossing edge, at a distance $w / 2$ of the eNB) while considering different reuse factors, $r_{c c}=D / R=2 K$. Although the first case also corresponds to a Manhattan grid, we label it as "Lin" (Linear) because the respective equations are formally the same as in the linear topology with cigar shaped cells. In the view chart, the number before "Man" or "Lin" represents $r_{c c} . C / I$ is higher when the user is at position 2 compared with the case where the UE is at position 1 (corresponding to the same equations for $C / I$ as in the linear topology). As the worst-case occurs if the user is at position 1 , we have considered the frequency reuse at distances $D-R$ and $D+R$, which in practice corresponds to considering the linear topology.

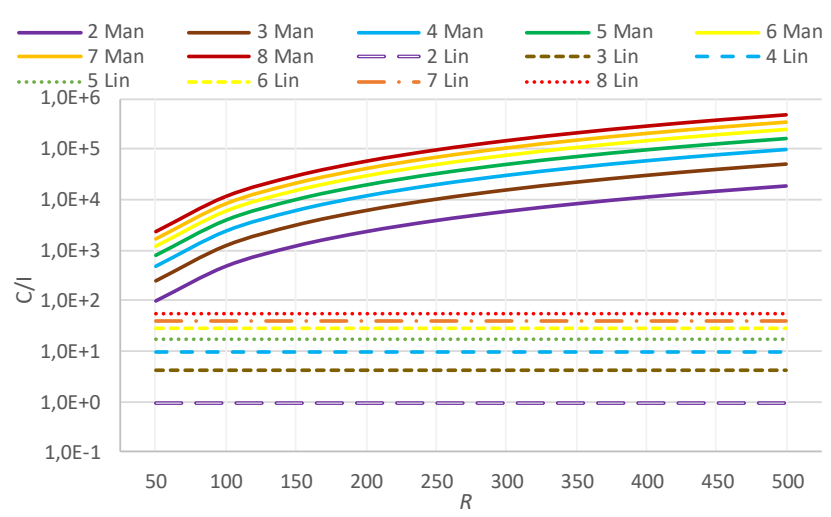

Fig. 3. Variation of $C / I$ with $R$, for the linear ("Lin") and Manhattan ("Man") topologies, while considering different reuse factors $\left(r_{c c}=D / R\right)$.

\section{CNIR AND PHYSICAL THROUGHPUT}

It is worthwhile to analyse the variation of the CNIR and Physical Throughput with the distance, $d(0 \leq d \leq R)$ while understanding the behaviour of the system equivalent throughout. The underlying implicit formulation (from [9]) maps the CNIR onto the values of the PHY supported throughput, $R_{b}$, through the corresponding MCS, as shown in Figure 4, where the index $J$ represents the MCS index. Figure 5 presents the variation of CNIR and $R_{b}$ with the distance, $d$, for $\sigma$ $\neq 0, R=50 \mathrm{~m}$ and different frequencies. The slight difference in the formulation consists on the fact that we are now considering linear cells in which the area is proportional to $R$; whereas in hexagonal cells (considered in [9]), the hexagonal crowns areas, corresponding to each individual MCSs, the area is proportional to $R^{2}$. Tables I and II show the parameters considered in the computations. The standard deviation $\sigma_{[\mathrm{db}]}$ is 0.004 for the 28 $\mathrm{GHz}$ frequency and 4.4 for 38,60 and $73 \mathrm{GHz}$.

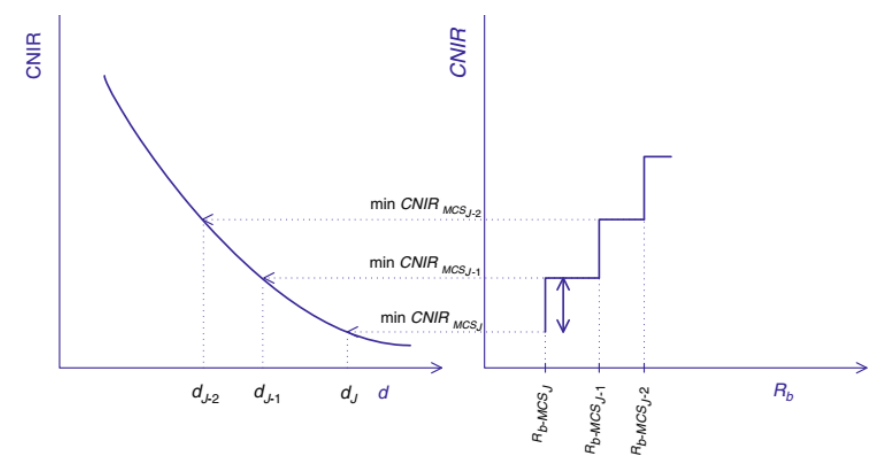

a) Correspondence of the PHY throughput for rings $J, J-1, J-2, \ldots$, with the minimum CNIRs of consecutive MCS that map to step distances $d_{J}, d_{J-1}, d_{J-2}, \ldots$

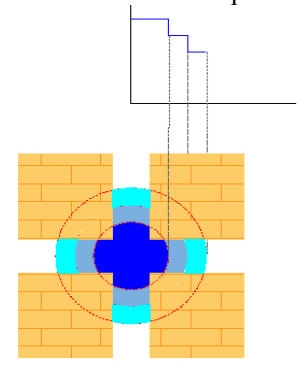

b) Areas of the coverage rings corresponding to a given PHY throughput. Fig. 4. Implicit formulation for the throughput (adapted from [8])). 


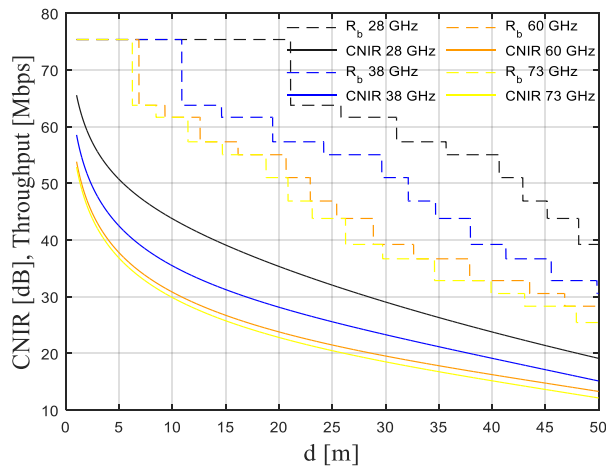

Fig. 5. Variation of the CNIR and throughput with $\mathrm{d}$ for $28 \mathrm{GHz}, 38 \mathrm{GHz}, 60$ $\mathrm{GHz}, 73 \mathrm{GHz}$, for $R=50 \mathrm{~m}$ for $\sigma \neq 0$.

TABLE I. PARAMETERS CONSIDERED IN THE ANALYSIS (FROM [4])

\begin{tabular}{|l|c|}
\hline Transmitter Power & $0 \mathrm{dBW}$ \\
\hline Transmitter gain & $3 \mathrm{dBi}$ \\
\hline Receiver gain & $0 \mathrm{dBi}$ \\
\hline Carrier & $20 \mathrm{MHz}$ \\
\hline Noise Figure & $7 \mathrm{~dB}$ \\
\hline Height (Base Station) & $7 \mathrm{~m}$ \\
\hline Height (User Equipment) & $1.5 \mathrm{~m}$ \\
\hline
\end{tabular}

TABLE II. VALUES FOR THE LOG-NORMAL RANDOM VARIABLE MINIMUM CNIR, MODULATION AND SPECTRAL EFFICIENCY VERSUS MCS, FOR LTE, AND VALUES FOR THE VERTICAL ASYMPTOTE FOR DL FROM 3GPP 36.213

\begin{tabular}{|c|c|c|c|c|c|}
\hline \multicolumn{5}{|c|}{ MCS mapping } & \multirow{2}{*}{$\frac{\boldsymbol{R}_{b|\mathrm{Mbps}|}}{20 \mathrm{MHz}}$} \\
\hline Mod. index & $\left|C N I R_{\min [\mathrm{dB}]}\right|$ & Modulation & ITBS & $S_{\text {eff }}$ & \\
\hline 1 & -4.63 & \multirow{10}{*}{ QPSK } & 0 & 0.23 & 2.797 \\
\hline 2 & -3.615 & & 1 & 0.31 & 3.624 \\
\hline 3 & -2.6 & & 2 & 0.38 & 4.584 \\
\hline 4 & -1.36 & & 3 & 0.49 & 5.736 \\
\hline 5 & -0.12 & & 4 & 0.6 & 7.224 \\
\hline 6 & 1.17 & & 5 & 0.74 & 8.76 \\
\hline 7 & 2.26 & & 6 & 0.88 & 10.296 \\
\hline 8 & 3.595 & & 7 & 1.03 & 12.216 \\
\hline 9 & 4.73 & & 8 & 1.18 & 14.112 \\
\hline 10 & 6.13 & & 9 & 1.33 & 15.84 \\
\hline 11 & 7.53 & \multirow{7}{*}{ 16-QAM } & 9 & 1.33 & 15.84 \\
\hline 12 & 8.1 & & 10 & 1.48 & 17.568 \\
\hline 13 & 8.67 & & 11 & 1.7 & 19.848 \\
\hline 14 & 9.995 & & 12 & 1.91 & 22.92 \\
\hline 15 & 11.32 & & 13 & 2.16 & 25.456 \\
\hline 16 & 12.78 & & 14 & 2.41 & 28.336 \\
\hline 17 & 14.24 & & 15 & 2.57 & 30.576 \\
\hline 18 & 14.725 & \multirow{12}{*}{ 64-QAM } & 15 & 2.57 & 30.576 \\
\hline 19 & 15.21 & & 16 & 2.73 & 32.856 \\
\hline 20 & 16.92 & & 17 & 3.03 & 36.696 \\
\hline 21 & 18.63 & & 18 & 3.32 & 39.232 \\
\hline 22 & 19.975 & & 19 & 3.61 & 43.816 \\
\hline 23 & 21.32 & & 20 & 3.9 & 46.888 \\
\hline 24 & 22.395 & & 21 & 4.21 & 51.024 \\
\hline 25 & 23.47 & & 22 & 4.52 & 55.056 \\
\hline 26 & 25.98 & & 23 & 4.82 & 57.336 \\
\hline 27 & 28.49 & & 24 & 5.12 & 61.664 \\
\hline 28 & 31.545 & & 25 & 5.33 & 63.776 \\
\hline 29 & 34.6 & & 26 & 5.55 & 75.376 \\
\hline
\end{tabular}

Results for $R=25$ and $100 \mathrm{~m}$ with $\sigma \neq 0$ and $\sigma=0$ are presented in [9]. It is worthwhile to note that the CNIR, hence $R_{b}$, decreases from the $28 \mathrm{GHz}$ to the $38 \mathrm{GHz}$ frequency band, and then to the 60 and $73 \mathrm{GHz}$ ones for coverage distances of up to circa $115 \mathrm{~m}$. Then, there is a trend for the relative behaviour between the $60 \mathrm{GHz}$ and $73 \mathrm{GHz}$ frequency band (due to the reduction of the coverage owing to the $\mathrm{O}_{2}$ attenuation excess), and the $60 \mathrm{GHz}$ frequency band will have worst cellular coverage than the $73 \mathrm{GHz}$ one.

\section{ANALYSIS OF PHY THROUGHPUT AND SYSTEM CAPACITY}

By applying the formulation for the computation of the equivalent supported throughput from [9], Chapter 9, p. 380, we obtain the curves for the supported throughput as a function of the coverage distance (not $d$ ) for the different frequency bands. We compare the cases where $R$ varies between 25 and $500 \mathrm{~m}$ with the case where $R$ varies between 5 and $100 \mathrm{~m}$. We consider LTE-U parameters adapted to millimetre waves.

In Figures 6 and 7, $R$ varies up to 100 and $500 \mathrm{~m}$, respectively, for $\sigma=0$ and $\sigma \neq 0$. In these curves, it is clear that, in general, the supported throughput is higher for the $28 \mathrm{GHz}$ frequency band compared to $38 \mathrm{GHz}$ but the $60 \mathrm{GHz}$ frequency band only performs better than the $73 \mathrm{GHz}$ band for $R \mathrm{~s}$ up to approximately $100 \mathrm{~m}$. Therefore, the throughput for the $73 \mathrm{GHz}$ frequency band is higher. This is due to the $\mathrm{O}_{2}$ attenuation excess which causes a reduction in the coverage at $60 \mathrm{GHz}$.

In order to study the influence of different frequency bands in the results for system capacity as a function of $R$, three dimensional (3D) graphs have been drawn, where the behaviour of the PHY throughput $\left(R_{b}\right)$ mapped into MCS (with 29 levels, in the $z z$ axis) is represented, as shown in Figures 8 and 9 $(R=100 \mathrm{~m})$. The cell size $(R)$ varies from $5 \mathrm{~m}$ to 100 . As in [10], [11], due to the range of distances $d$ is different while cell radii vary from $(0 \leq d \leq R)$, in the view charts we have considered the normalized distance, defined as $d / R$, when representing the stepwise behaviour of the PHY throughput that defines the ring area which is using a certain MCS within the cell.

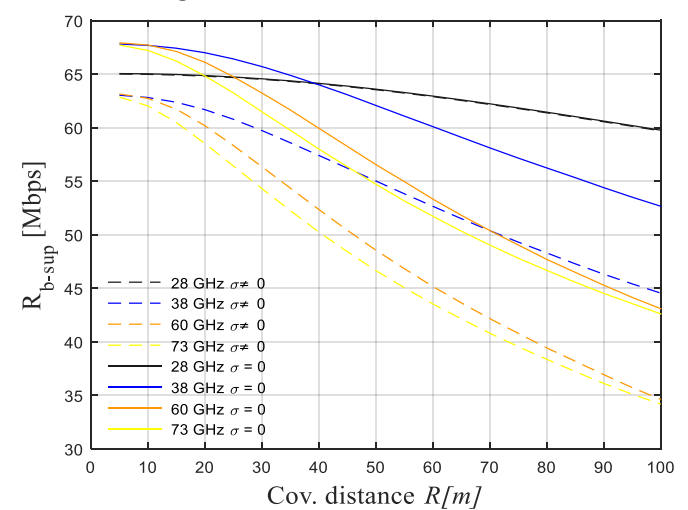

Fig. 6. Supported throughput for $28 \mathrm{GHz}, 38 \mathrm{GHz}, 60 \mathrm{GHz}, 73 \mathrm{GHz}$, and $R=100$ $\mathrm{m}$, for $\sigma=0$ and $\sigma \neq 0$.

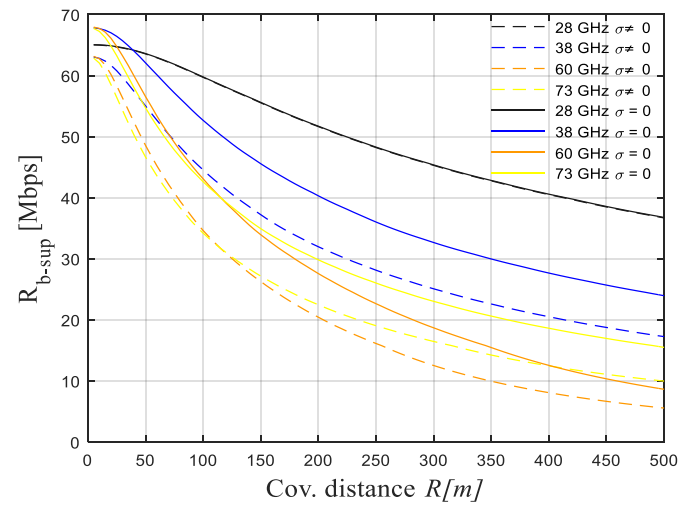

Fig. 7. Supported throughput for $28 \mathrm{GHz}, 38 \mathrm{GHz}, 60 \mathrm{GHz}, 73 \mathrm{GHz}$, and $R=500$ $\mathrm{m}$, for $\sigma=0$ and $\sigma \neq 0$. 


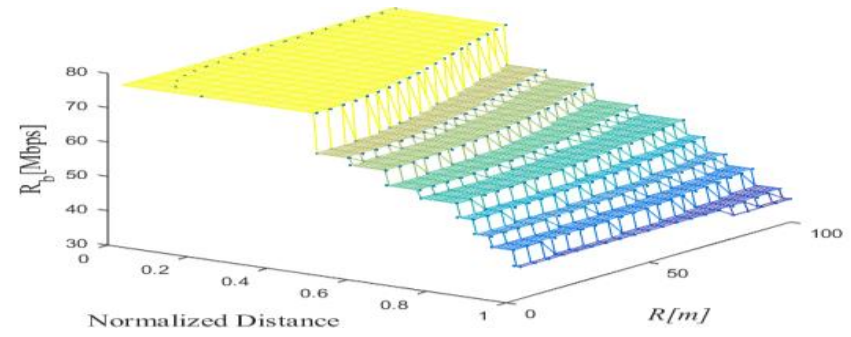

Fig. 8. 3D view graph for the PHY throughput mapped into MCS (with 29 levels, in the $z z$ axis), for $\sigma=0,28 \mathrm{GHz}$ and $R=100 \mathrm{~m}$.

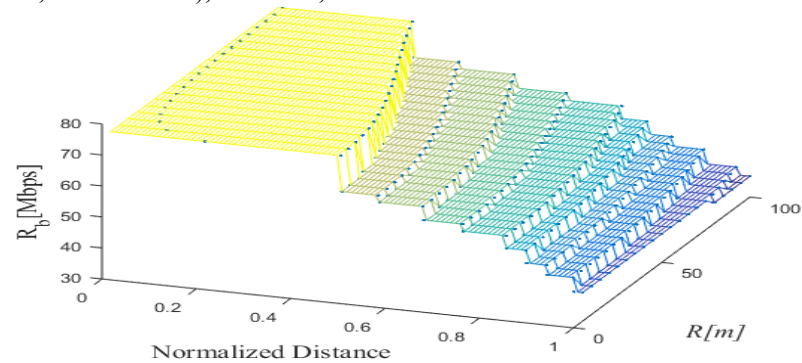

Fig. 9. 3D view graph for the PHY throughput mapped into MCS (with 29 levels, in the $z z$ axis), for $\sigma \neq 0,28 \mathrm{GHz}$ and $R=100 \mathrm{~m}$.

The normalized distance represents the variation of $d$ from 0 to $R$. The cell ranges are represented in the $y y$ axis while the normalized distance is shown in the $x x$ axis and varies from 0 to 1 . By analysing these surfaces of the PHY throughput, it can be understood that the decrease in the MCSs, while the distance varies inside the cell, is faster for longer $R$ s and an interpretation of the behaviour for the different frequency bands is possible. Results are clearly better for $\sigma=0$ compared to the case $\sigma \neq 0$ (when the fading margin is considered for coverage purposes).

\section{COST/REVENUE TRADE-OFF}

Concerning to the enhancement of the system from the profit point of view, we compute from throughput the results for cost and revenues, using the model described in [12], [13]. The scenario presented in Figure 4, behaves similarly to a Manhattan geometry outdoor environment, through the respective linearization described in Section I and Figure 1, with omni-directional cells and antennas.

The overall cost of the network per unit length, per year, $C_{0[\epsilon / u l]}$, is given by

$$
C_{0[€ / u l]}=C_{f i[€ / u l]}+C_{f b} \cdot N_{c / u l}
$$

where $C_{f i}$ is a fixed term cost, $C_{b f}$ is cost proportional to the number of $B S s$, and the number of cells per unit area is given by

$$
N_{C / u l}=\frac{1}{2 \cdot R_{[k m]}-\frac{w_{[k m]}}{2}}
$$

It is worthwhile to note that the building block dimensions change with $R$, since the side length is $(2 R-w)$, causing a variation in the streets area, what does not occur in reality. However, the linearized curves of costs and profits of the network, shown in Figures 10 to 11 are not affected, since only the street length is considered, instead of the area [12], [13]

The revenues per cell, $\left(R_{v}\right)_{/ c e l l[\epsilon]}$, can be attained as a function of the throughput per Base Station, $B S$, $t h r_{B S[\mathrm{kbps}]}$, and the revenue of a channel with a data rate $R_{b[\mathrm{kbps}],} R_{r b[\epsilon / \mathrm{min}]}$, and $T_{b h}$ corresponding the equivalent duration of busy hours per day [12], $R_{v / c e l l[€]}$ can be obtained by

$$
\left(R_{v}\right) \operatorname{cell}[€]=\frac{\left.\operatorname{thr}_{B S[k b p s]} \cdot T_{b h} \cdot R_{r b[€ / m i n}\right]}{R_{b[k b p s]}}
$$

Revenues are considered in annual basis, where we considered six busy hours per day, 240 busy days per year [10], and the price of a $144 \mathrm{kbps}$ "channel" per minute (corresponding to the price of $\approx 1 \mathrm{MB}$ ), considering $R_{144}$ $\mathrm{kbps}[\epsilon / \mathrm{min}]=0.07$. Considering the throughput with $\sigma \neq 0$ in order to obtain more realistic values. The revenue per cell can be obtained by

$$
\left(R_{v}\right) \operatorname{cell}[€]=\frac{\operatorname{thr}_{B S[k b p s]} \cdot 60 \cdot 6 \cdot 240 \cdot R_{r b[€ / \mathrm{min}]}}{144_{[\mathrm{kbps}]}}
$$

Figure 12 presents results for the revenue per cell per year, with $R_{b}=144 \mathrm{kbps}$, through variation of $R$ for $R_{\max }=100 \mathrm{~m}$. It is evident that $28 \mathrm{GHz}$ band is the most profitable of all bands for all distances and the profit will be lower as the distance increases for all frequencies.

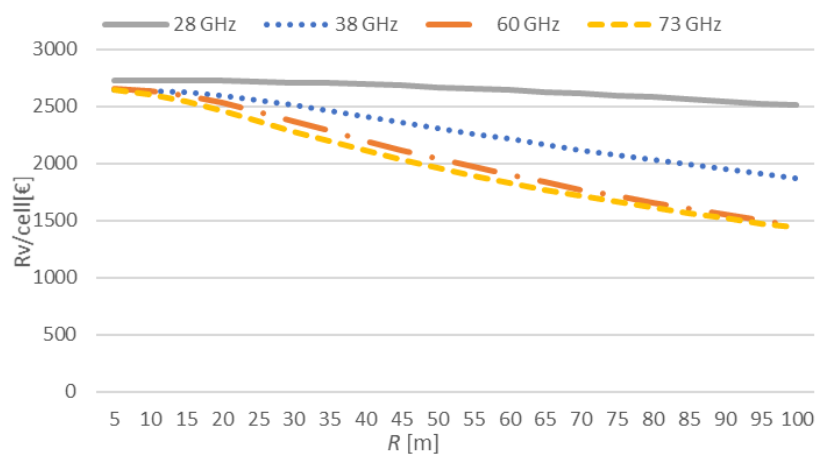

Fig. 10. Revenue per cell with $R_{b}=144 \mathrm{kbps}$ and $5 \leq R \leq 100 \mathrm{~m}$.

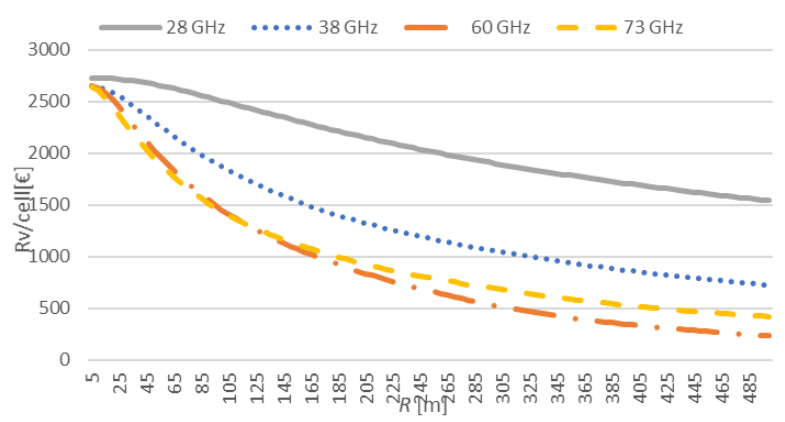

Fig. 11. Revenue per cell with $R_{b}=144 \mathrm{kbps}$ and $5 \leq R \leq 500 \mathrm{~m}$.

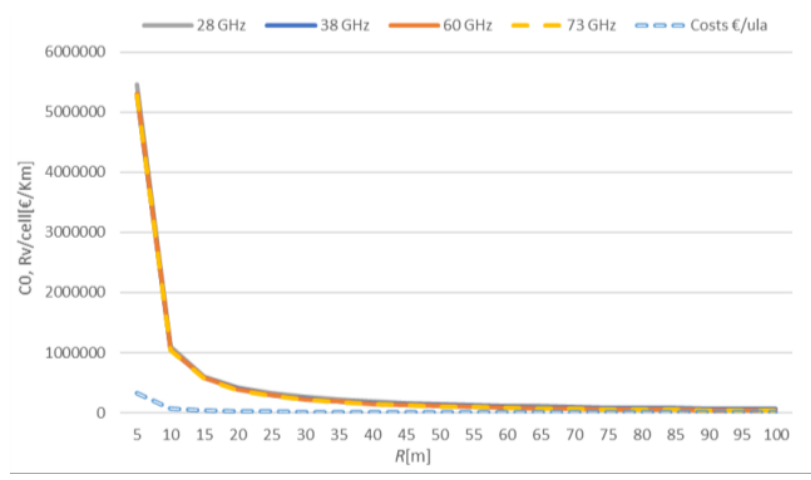

Fig. 12. Network revenue/cost per unit length per year as a function of $R$, with $R_{\max }=100 \mathrm{~m}$. 
The revenue per unit area per year, $R_{v}$, is obtained multiplying the revenue per cell by the number of cells per unit of area, as follows

$$
\begin{gathered}
R_{v[€ / \mathrm{km}]}=N_{c / u l a} \cdot\left(R_{v}\right) \operatorname{cell}[€] \\
R_{v[€ / \mathrm{ula}]}=\frac{1}{2 \cdot R_{[\mathrm{km}]}-\frac{w_{[\mathrm{km}]}}{2}} \cdot \frac{\operatorname{thr}_{B S[\mathrm{kbps}] \cdot T_{b h} \cdot R_{r b[€ / \mathrm{min}]}}}{R_{b[\mathrm{kbps}]}}
\end{gathered}
$$

$C_{f b}$ is given by

$$
C_{f b[€]}=\frac{C_{B S}+C_{\text {Inst }}}{N_{\text {year }}}+C_{M \& O}
$$

$C_{f b}$ can be calculated by assumption present in Table III, for five-year project duration, assuming unlicensed bands.

In a near future, with the equipment normalization and mass production, the equipment prices will get lower, making this kind of communication more accessible comparable to the modem "normal prices", like "Qualcomm Snapdragon X50" modem. Qualcomm, according to the "Snapdragon X50 5G" modem is capable of download speeds of up to $5 \mathrm{Gbps}$ which is five times faster than the fastest $4 \mathrm{G}$ modem [14], [15].

The profit, $P_{f t}$, is an important outcome to enhance the network, and is given by the difference between the revenues and the costs in $€ / \mathrm{km}$, while the profit in percentage is given by the net revenue normalized by the cost

$$
P_{f t[€ / \mathrm{km}]}=\left(\left(R_{v}\right)_{[€ / \mathrm{km}]}-C_{0[€ / \mathrm{km}]}\right) / C_{0[€ / \mathrm{km}]}
$$

We consider the profit in percentage instead of the absolute profit because this metric is more relevant for operators and service providers [13]. If $R_{v}[\epsilon / \mathrm{km}]-C_{0[\epsilon / \mathrm{km}]}$ is positive, there will be profit. Figures 13 and 14 present the results for the overall cost per unit length per year, $C_{0}$, and the revenue per unit length per year, for only one carrier. For our assumptions, the revenues are noticeably higher than the costs of all frequencies. Some curves are overlapped showing similar revenues. Considering all the computation with the respective price per minute and all the costs from Table III, for 60 and $73 \mathrm{GHz}$ frequency bands, for distances longer than $210 \mathrm{~m}$ and $245 \mathrm{~m}$, respectively, and for $38 \mathrm{GHz}$ frequency band with distances above $440 \mathrm{~m}$, the system is not profitable. For all studied distances, $28 \mathrm{GHz}$ is the only profitable frequency band for the longest coverage distances. For distances between $5 \mathrm{~m}$ and 20 $m$ the profit is higher than $240 \%$ at $28 \mathrm{GHz}$ and $220 \%$ for the remaining frequencies.

For the 60 and $73 \mathrm{GHz}$ bands, as shown in Figures 17 to 18, the average profit is similar for all range of $R s$, while for distances longer than $130 \mathrm{~m}$ the profit for the $73 \mathrm{GHz}$ band is higher due to the highest system capacity (as the extra $\mathrm{O}_{2}$ attenuation additionally affects coverage at $60 \mathrm{GHz}$ ).

TABLE III. ASSUMPTIONS FOR BASE STATION COSTS FROM [12]

\begin{tabular}{|l|c|}
\hline Parameters & Values $[\boldsymbol{\epsilon}]$ \\
\hline Initial costs: & 2500 \\
BS price, $C_{B S}$ & 250 \\
Installation, $C_{\text {Inst }}$ & 0 , unlicensed bands \\
Fixed, $C_{f i}$ & \\
Annual Cost: & 250 \\
Operation and & \\
maintenance, $C_{M \& O}$ & \\
\hline
\end{tabular}

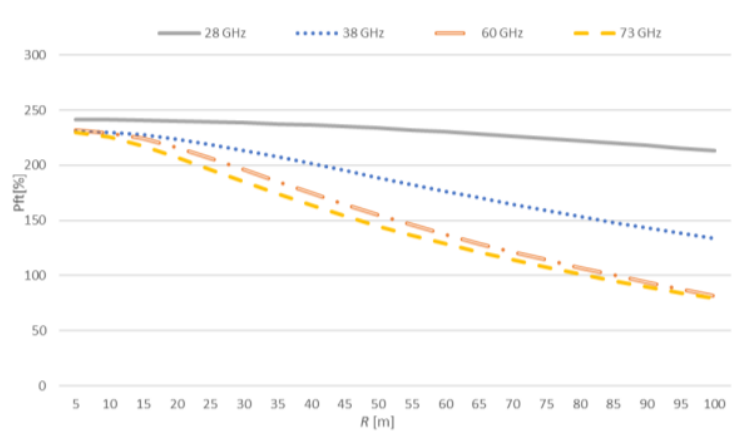

Fig. 13. - Profit per unit length per year, in percentage with $R_{\max }=100 \mathrm{~m}$.

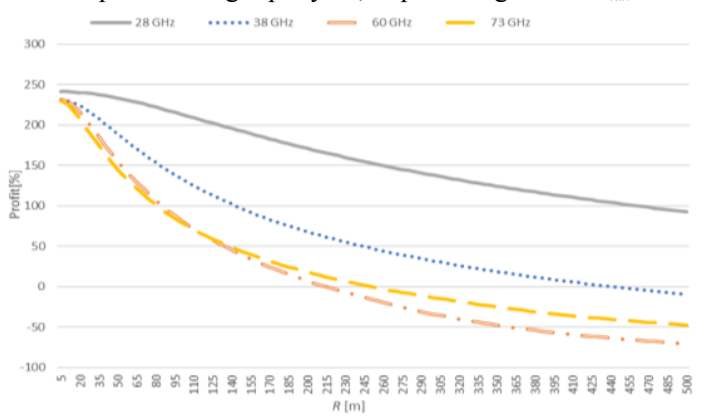

Fig. 14 - Profit per unit length per year, in percentage with $R_{\max }=500 \mathrm{~m}$.

The results clearly indicate that coverage distances up to 80 $\mathrm{m}$ should be used to optimise the system by having into consideration the maximization of the profit in percentage. As the goal of operators and service providers is to enhance the profit, where the profitable zone is for short distances mainly in the $28 \mathrm{GHz}$ frequency band, from 5 to $80 \mathrm{~m}$.

\section{CONCLUSION}

In this study, we identify and discuss the potentialities of mobile cellular communications in the millimetre wavebands, showing that very high bit/data rates can be supported in small cells with short-range coverage. As in real environments, cellular mobile communications are simultaneously affected by noise and co-channel interference, we have studied the behaviour of the carrier-to-noise-plus-interference ratio with the coverage distance (and the actual distance from users to the eNBs) while analysing its impact in cellular planning and the optimization process. Foremost, for $\gamma=2.3$ (40 and $60 \mathrm{GHz}$ ), the carrier-to-interference ratio $(C / I)$ is increasing with $R$ and is clearly higher when the co-channel reuse factor $r_{c c}=D / R$ raises from 2 to $8 . C / I$ is higher when the user is at position 2 compared with the case where the UE is at position 1 corresponding to the worst-case in the linear topology.

The equivalent supported throughput within cells was assessed while considering reuse pattern $K=2$, assuming in this preliminary phase that LTE is considered, but other air interfaces will also be assumed. In terms of cell coverage and the computation of interference, LoS propagation models have been considered at the 28, 38, 60 and $73 \mathrm{GHz}$ bands.

From these analytical computations, we have learned that the highest system capacity and the highest modulation coding schemes are achieved for coverage distances up to $30 \mathrm{~m}$. Likewise the supported throughput decreases for the longest coverage ranges and it is clearly more favourable for the lowest 
frequency bands. However, in terms of interference the system shows highest interference for lowest frequency bands.

For $\sigma=0$, the 38,60 and $73 \mathrm{GHz}$ frequency bands only performs better than $28 \mathrm{GHz}$ frequency band for $R s$ up to $20 \mathrm{~m}$, $25 \mathrm{~m}, 38 \mathrm{~m}$ for, 73, 60 and $38 \mathrm{GHz}$ respectively as shown Figure 15. For $\sigma \neq 0$, In general, the supported throughput is higher for the $28 \mathrm{GHz}$ frequency band, which always becomes higher than the values for the other frequency bands, $\left(\sigma_{[\mathrm{dB}]}=0.004\right.$ at 28 $\mathrm{GHz}$ and $\sigma_{[\mathrm{dB}]}=4.4$ for at 38,60 and $73 \mathrm{GHz}$ ) for the whole range of coverage distances, showing that the better the coverage is the higher the system capacity becomes, although the system capacity is inferior for $\sigma \neq 0$.

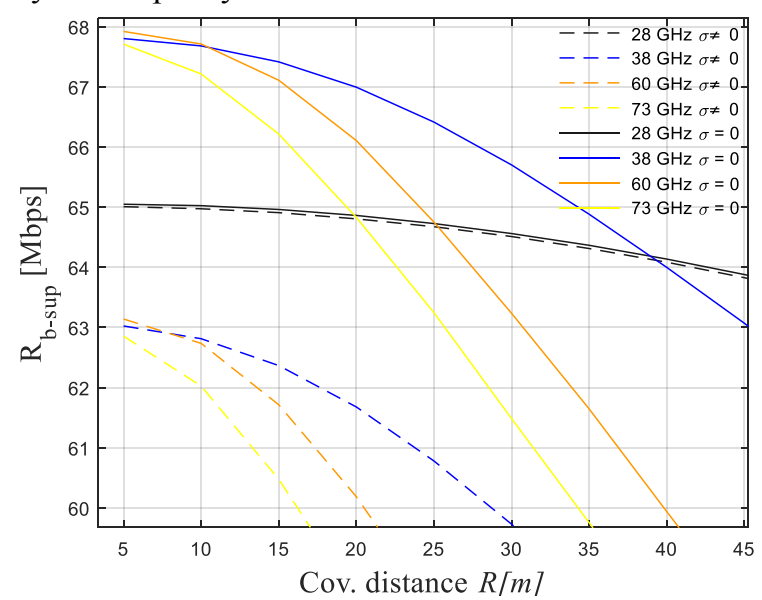

Fig. 15 - Zoomed plot from figure 7, Supported throughput for $28 \mathrm{GHz}, 38$ $\mathrm{GHz}, 60 \mathrm{GHz}, 73 \mathrm{GHz}$, for $\sigma=0$ and $\sigma \neq 0$.

The $60 \mathrm{GHz}$ frequency band only performs better than the $73 \mathrm{GHz}$ band for $R \mathrm{~s}$ up to approximately $115 \mathrm{~m}$. After this distance, the throughput for the $73 \mathrm{GHz}$ frequency band is higher. This fact is due to the $\mathrm{O}_{2}$ attenuation excess which results in the reduction of the coverage at $60 \mathrm{GHz}$, yielding lower values for the supported throughput.

In our scenario, the profit is mostly a decreasing function with $R$. Consequently, the most profitable cell radii are the lowest ones for all frequencies, and the best behaviour occurs in the $28 \mathrm{GHz}$ band. For long distances, the system ceases to be profitable. Revenues are proportional to the supported throughput. Computations show that, in the future, it is possible to install these types of structure when costs of installation and maintenance the network decreases, enabling higher system capacity while reducing prices. The optimum values for the profit in percentage are obtained in the range $R \approx 5 \mathrm{~m}$ to $R \approx$ $20 \mathrm{~m}$, with profit near to $240 \%$. The results clearly indicate that coverage distances up to $80 \mathrm{~m}$ should be used to optimise the system in terms of profit.

\section{ACKNOWLEDGMENT}

Authors would like to thank Sofia Sousa and Rui Paulo for the unconditional support.

\section{REFERENCES}

[1] F. J. Velez, M. Dinis and J. Fernandes, "Mobile Broadband Systems: Research and Visions," IEEE VTS (Vehicular Technology Society) News, Vol. 52, No.2, May 2005.
[2] T.S. Rappaport et al., "Millimeter Wave Mobile Communications for 5G Cellular: It Will Work!", IEEE Access, vol. 1., pp. 335-349, May 2013.

[3] J.M. Brázio and F.J. Velez, "Design of Cell Size and Frequency Reuse for a Millimetrewave Highway Coverage Cellular Communication System," in Proc. of PIMRC'96 - $7^{\text {th }}$ IEEE International Symposium on Personal Indoor, and Mobile Communications, Taipei, Taiwan, ROC, Oct. 1996.

[4] T. S. Rappaport, R. W. Heath and R. Daniels, and J. Murdock, Millimeter wave wireless communications, Prentice Hall, Pearson Education, Upper Saddle River, NJ, USA, 2014.

[5] M. K. Samimi, T. S. Rappaport, and G. R. MacCartney, "Probabilistic omnidirectional path loss models for millimeter-wave outdoor communications," IEEE Wireless Communicat. Letters, vol. 4, no. 4, Aug. 2015, pp. 357-360.

[6] A. I. Sulyman, A. T. Nassar, M. K. Samimi, G. R. Maccartney, T. S. Rappaport and A. Alsanie, "Radio propagation path loss models for $5 \mathrm{G}$ cellular networks in the $28 \mathrm{GHz}$ and $38 \mathrm{GHz}$ millimeter-wave bands," IEEE Communicat. Magazine, vol. 52, no. 9, Sept. 2014., pp.78-86

[7] T. S. Rappaport, G. R. MacCartney, M. K. Samimi and S. Sun, "Wideband Millimeter-Wave Propagation Measurements and Channel Models for Future Wireless Communication System Design," IEEE Transactions on Communications, vol. 63, no. 9, Sept. 2015, pp. 3029-3056.

[8] F. J. Velez and L. M. Correia, J. M. Brázio, "Frequency Reuse and System Capacity in Mobile Broadband Systems: Comparison between the 40 and $60 \mathrm{GHz}$ Bands," Wireless Personal Communications, vol.19, no. 1, Aug. 2001, pp.1-24.

[9] E. S. B. Teixeira, S. Sousa, R. R. Paulo and F. J. Velez, "Frequency Reuse Trade-off and System Capacity in Small Cell Networks in the Millimetre Wavebands," $3^{\text {rd }}$ Meeting of the Management Committee of COST CA 15104 - IRACON, TD(17)04084, Lund, Sweden, May 2017.

[10]R. Prasad and F. J. Velez, WiMAX Networks: Technoeconomic Vision and Challenges, Springer, Dordrecht, The Netherlands, 2010.

[11]S. Sousa, F. J. Velez, J. M. Peha, "Impact of considering the ITU-R Two Slope Propagation Model in the System Capacity Trade-off for LTE-A HetNets with Small cells," in Proc. of 32nd URSI GASS 2017, Montreal, Canada, Aug. 2017.

[12]F. J. Velez, and L. M. Correia. "Optimisation Of Mobile Broadband Multi-Service Systems Based In Economics Aspects," Wireless Nets. vol.9, no. 5, Sep 2003: pp. 525-533.

[13]F. J. Velez, O. Cabral, F. Merca, V. Vasos. Service characterization for cost/benefit optimization of enhanced UMTS. Telecom. Systems vol. 50, no. 1, Apr 2012, pp 31-45.

[14]J. Gozalvez, "5G Worldwide Developments [Mobile Radio]," in IEEE Vehicular Technology Magazine, vol. 12, no. 1, March 2017, pp. 4-11.

[15]Qualcomm.(2017, October). Networks (2nd ed.) [Online]. Available:https://www.qualcomm.com/products/snapdragon/ modems $/ 5 \mathrm{~g} / \mathrm{x} 5$

[16]Forbes(2017,October).[Online].https://www.forbes.com/sites /patrickmoorhead/2016/10/17/qualcomm-unveils-theworlds-first-5g-modem-snapdragon-x50-for-28-ghzmmwave-networks/\#b2c5f655a670 